\title{
La vacunación antineumocóccica en adultos mayores: certezas e incertidumbres
}

Pneumococcal vaccination in elderly adults: certainties and uncertainties

Juan Victor Ariel Franco $\$$

Franco J. La vacunación antineumocóccica en adultos mayores: certezas e incertidumbres. Evid Act Pract Ambul. 2017;20(1):2-3.

En el presente número de Evidencia se presenta el comentario sobre el ensayo clínico CAPITA donde se evaluó la eficacia de la vacuna antineumocóccica conjugada 13-valente (VC13) en adultos mayores a 65 años ${ }^{1}$. En este estudio se observó una menor incidencia de enfermedad invasiva y no invasiva por neumococo. Estos resultados son los primeros de un estudio que evalúa desenlaces clínicos para la vacuna conjugada, dado que hasta ahora se contaba con varios estudios que evaluaban resultados intermedios (subrogados), como la inmunogenicidad.

Las vacunas conjugadas habían sido desarrolladas inicialmente para la generación de inmunidad ante organismos capsulados (S. pneumoniae, $\mathrm{H}$. influenzae, N. meningitidis) en individuos menores a dos años. Esto se debe a que esta población tiene una susceptibilidad mayor a sufrir infecciones invasivas por estos gérmenes debido a la pobre respuesta inmunitaria a antígenos polisacáridos (de la cápsula) por la falta de del desarrollo de las células B de la zona marginal del bazo. La "conjugación" del antígeno polisacárido con una proteína, permite la aparición de una respuesta mediada por células B2. Estas células generan anticuerpos para antígenos peptídicos e inmunidad contra antígenos virales y bacterianos en respuesta a otras vacunas (ej. para difteria, tétanos, etc. $)^{2}$. Conceptualmente, una vez desarrollada la inmunidad de las células B de la zona marginal del bazo, los adultos pueden generar una respuesta inmune adecuada para gérmenes capsulados ${ }^{3}$. Uno de los beneficios de la vacunación antineumocócica en niños es la inmunidad de rebaño: por la menor circulación de la bacteria, se producen menos infecciones en individuos no vacunados, especialmente adultos ${ }^{4}$. Hasta el 2016, las recomendaciones de las sociedades científicas y del Ministerio de Salud de la Nación Argentina incluían la vacunación con la vacuna polisacárida 23-valente no conjugada $(\text { VP23 })^{5}$ en base a la evidencia disponible en relación a la reducción de enfermedad invasiva por neumococo $0^{6,7,8,9}$. A raíz de los resultados del estudio CAPITA sobre la eficacia de la VC13 ${ }^{10}$, la evidencia de sinergia inmunológica entre la inmunización con vacuna conjugada y no conjugada ${ }^{11}$ y diversos estudios que modelaron la costoefectividad de los esquemas secuenciales utilizando ambas vacunas ${ }^{12}$, el Comité de Inmunizaciones del Centro de Control de Enfermedades de Estados Unidos (en inglés Advisory Committee on Inmunization Practice, ACIP) emitió en 2014 la recomendación del uso de la VC13 en adultos seguida de la vacunación por VP23 con un intervalo de 6 a 12 meses $^{13}$. Este año, el Ministerio de Salud de la Nación Argentina, lanzó un programa de inmunización para neumococo en mayores de 65 años que incluye el mismo esquema recomendado por la ACIP en Estados Unidos con el objetivo de lograr una inmunización mayor al $95 \%$ de la población diana ${ }^{14}$.
A la hora de incorporar una vacuna en el calendario se deben tener algunos aspectos de utilidad ${ }^{15}$. Ver Tabla 1.

Tabla 1. Aspectos a considerar a la hora de incluir una vacuna en el calendario.

\section{1) Carga de enfermedad}

2) Características de la nueva vacuna y su impacto en la factibilidad del programa [de vacunación]

3) Percepción general del riesgo [de la enfermedad]

4) Disponibilidad de las vacunas [a incorporar]

5) Intervenciones (alternativas) y análisis económico

6) Compromiso y apoyo político

7) Alianza con diversos sectores [involucrados en el programa de vacunación]

Adaptado de Augustovski F. ${ }^{15}$

La enfermedad invasiva y no invasiva por neumococo es un problema de salud que cumple con los requisitos $1,3,6$ y 7 , tanto por su morbimortalidad como por el acuerdo de la sociedad a la hora de priorizarlo como problema de salud. A su vez, las vacunas disponibles son accesibles y seguras, por los cuales se cumplirían los requisitos 2 y 4 .

Sin embargo, una de las dificultades que nos plantea la decisión de incorporar la vacuna antineumocóccica es evaluar las diferentes alternativas, su eficacia y efectividad relativa y su impacto económico (punto 5 de la Tabla 1). Si bien ambas vacunas (VC13 y VP23) han sido eficaces en reducir la enfermedad por neumococo ("certezas"), la evidencia de la eficacia clínica directa de su sinergia en un esquema secuencial no ha sido probada en ensayos clínicos ("incertidumbres") y sólo hay disponibles estudios de inmunogenicidad (ver gráfico 1$)^{16}$. A su vez, existe evidencia de eficacia clínica tanto para la VP23 (al menos 18 ensayos clínicos con 64.901 pacientes ${ }^{17}$ ) como para la VC13 (estudio CAPITA con 84.496 pacientes) pero no existirían ensayos clínicos comparativos entre las vacunas ("incertidumbres"). EI estudio CAPITA a pesar de su gran magnitud, registró 90 y 49 casos de neumonía bacteriana confirmada en sus ramas placebo y experimental respectivamente, por lo cual la magnitud del efecto absoluto, a simple vista parece modesta. No se han demostrado cambios en la mortalidad. Se ha sugerido que los resultados de este estudio serían sobreestimados actualmente dado que, en Países Bajos, donde se realizó el estudio, se había implementado la vacunación en niños recientemente y no había posibilidad aún de inmunidad de rebaño ${ }^{18}$. Es importante destacar que actualmente el costo de la dosis de VC13 es aproximadamente el doble del de la VP23 $3^{19,20}$.

Figura 1. Evidencia directa e indirecta de eficacia de la vacunación con vacuna conjugada 13-valente (VC13) y vacuna polisacárida 23-valente (VP23).

EVIDENCIA DIRECTA

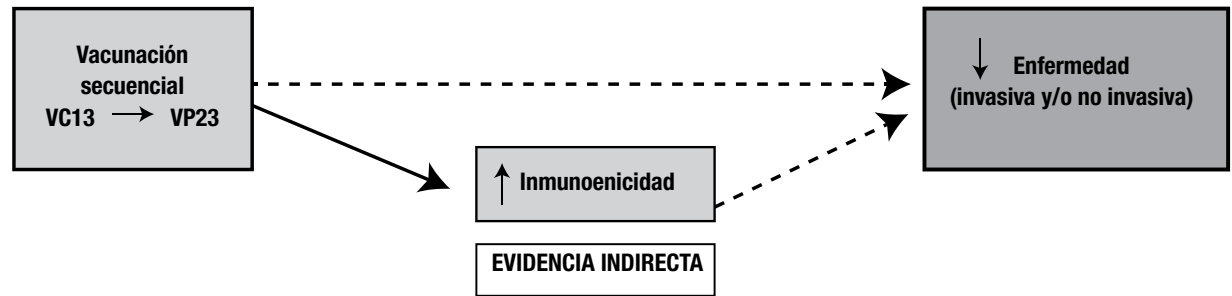

§ Servicio de Medicina Familiar y Comunitaria y Centro Cochrane Argentino Instituto Universitario del Hospital Italiano de Buenos Aires. juan.franco@ hospitalitaliano.org.ar 
Ante este escenario existirían al menos tres alternativas en términos de estrategias de vacunación:

1) Mantener esquema de vacunación VP23: basado en la evidencia científica directa disponible acerca de la eficacia clínica (status quo).

2) Cambiar a un esquema de vacunación basado en VC13: basado en la evidencia científica directa disponible acerca de la eficacia clínica. Teniendo en cuenta que es una vacuna de mayor costo (aproximadamente el doble), esta decisión debería estar apoyada por una evaluación económica tanto de costoefectividad como de impacto presupuestario.

3) Cambiar a un esquema secuencial basado en VC13 seguida de VP23: basado en evidencia científica indirecta de su sinergia (inmunogenicidad). Al tener en cuenta que con respecto al status quo, esto implica al menos una triplicación en los costos de implementación, esta decisión debería estar apoyada por una evaluación tanto de costoefectividad como de impacto presupuestario. El Ministerio de Salud Argentino ha optado por esta última opción, si bien el informe técnico no incluye datos económicos.
Como médicos de atención primaria respetamos el marco regulatorio de nuestro país que establece la obligatoriedad de las vacunas de los programas nacionales (Art 11. Ley 22.909) ${ }^{21}$ y las consecuencias relacionadas a su no aplicación, en formas de multas o vacunación compulsiva (Art 17 y 18 de la misma ley). La obligatoriedad se ve acompañada de la responsabilidad del Estado en la provisión gratuita de las vacunas (en este caso VC13 y VP23), lo cual se estaría cumpliendo en la Ciudad de Buenos Aires, según lo pudimos comprobar. Entendemos también que este marco regulatorio ha sido útil en el logro de altas tasas de vacunación y reducción y hasta erradicación de enfermedades inmunoprevenibles ${ }^{22}$. Sin embargo, tal como se plantea en este artículo, hay aspectos críticos a reflexionar en relación a la certidumbre de la evidencia científica que brinda apoyo a la toma de decisiones. Esperamos que futuras investigaciones provean información para tener mayor confianza en los efectos de las decisiones que hemos tomado como país.

Recibido 15/05/2017 y aceptado el 20/05/2017

\section{Referencias}

1. Nemirovsky C. Vacuna polisacárida conjugada contra la neumonía neumocócica en adultos. Evid. Act Pract Ambul. 2017;20(1):xx. Comentario de: Bonten MJM, y col. Polysaccharide Conjugate Vaccine against Pneumociccal Pneumonia in Adults: a randomided controlled trial. N Engl J Med 2015;372:1114-25. PMID: 25785969.

2. Principi N, y col. Prevention of Community-Acquired Pneumonia with Available Pneumococcal Vaccines. Int J Mol Sci. 2016 Dec 25;18(1). pii: E30. doi: 10.3390/ijms18010030. 3. Simonsen V, y col. Immunogenicity of a 23-valent pneumococcal polysaccharide vaccine in Brazilian elderly. Braz J Med Biol Res. 2005;38(2):251-260.

4. Westerink MA, y col Immune Responses to pneumococcal vaccines in children and adults: Rationale for age-specific vaccination. Aging Dis. 2012 Feb;3(1):51-67.

5. Sociedad Argentina de Infectología. Vacunación contra Neumococo en Adultos - Situación Actual en Argentina 2012 [Internet: fecha de último acceso 02/06/17]. Disponible en: https://www.sadi.org.ar/institucional/comisiones-de-trabajo/publicaciones-de-las-comisiones/item/174-documento-sobre-vacuna-antineumococica

6. Falkenhorst G, y col. Effectiveness of the 23-Valent Pneumococcal Polysaccharide Vaccine (PPV23) against Pneumococcal Disease in the Elderly: Systematic Review and Meta-Analysis. PLoS One. 2017 Jan 6;12(1):e0169368. doi: 10.1371/journal.pone.0169368. eCollection 2017.

7. Diao WQ, y col. Efficacy of 23-valent pneumococcal polysaccharide vaccine in preventing community-acquired pneumonia among immunocompetent adults: A systematic review and meta-analysis of randomized trials. Vaccine. 2016 Mar 18;34(13):1496-503. doi: 10.1016/j.vaccine.2016.02.023. Epub 2016 Feb 17. Review. PubMed PMID: 26899376. 8. Kraicer-Melamed $\mathrm{H}$, y col. The effectiveness of pneumococcal polysaccharide vaccine 23 (PPV23) in the general population of 50 years of age and older: A systematic review and meta-analysis. Vaccine. 2016 Mar 18;34(13):1540-50. doi: 10.1016/j.vaccine.2016.02.024.

9. Schiffner-Rohe J, y col. Efficacy of PPV23 in Preventing Pneumococcal Pneumonia in Adults at Increased Risk-A Systematic Review and Meta-Analysis. PLoS One. 2016 Jan 9. Schiffner-Rohe J, y col. Efficacy of PPV23 in Preventing Pneumococcal Pneumon

10. Bonten MJ, y col. Polysaccharide conjugate vaccine against pneumococcal pneumonia in adults. N Engl J Med. 2015 Mar 19;372(12):1114-25. doi: 10.1056/NEJMoa1408544 11. Greenberg RN, y col. Sequential administration of 13-valent pneumococcal conjugate vaccine and 23 -valent pneumococcal polysaccharide vaccine in pneumococcal vaccinenaïve adults 60-64 years of age. Vaccine. 2014 Apr 25;32(20):2364-74. doi: 10.1016/j.vaccine.2014.02.002. Epub 2014 Mar 5.

12. Chen J, y col. Cost-effectiveness of pneumococcal vaccines for adults in the United States. Adv Ther. 2014 Apr;31(4):392-409. doi: 10.1007/s12325-014-0115-y. Epub 2014 Apr 10.

13. Tomczyk S, y col. Use of PCV-13 and PPSV-23 vaccine among adults aged 65 and older: recommendations of the ACIP. MMWR. 2014;63(37);822-5.

14. Ministerio de Salud. Vacunación Contra Neumococo. Lineamientos Técnicos. Manual del Vacunador. Estrategia Argentina $2017-2018$ [Internet: fecha de último acceso 02/06/17]. Disponible en: http://www.msal.gob.ar/images/stories/bes/graficos/0000000947cnt-Lineamientos_neumo_adultos_WEB.pdf

15. Augustovski F, y col. Toma de decisiones acerca de incorporación de nuevas vacunas. Evid Pract Ambul 2011. 14(3) 82-4

16. Puhan MA, y col; GRADE Working Group. A GRADE Working Group approach for rating the quality of treatment effect estimates from network meta-analysis. BMJ. 2014 Sep 24;349:g5630. doi: 10.1136/bmj.g5630. Erratum in: BMJ. 2015;350:h3326.

17. Moberley S, y col. Vaccines for preventing pneumococcal infection in adults. Cochrane Database of Systematic Reviews 2013, Issue 1. Art. No.: CD000422. DOI: $10.1002 / 14651858 . C D 000422$ pub3.

18. Hochman M, y col. Reconsidering Guidelines on the Use of Pneumococcal Vaccines in Adults 65 Years or Older. JAMA Intern Med. 2015 Dec;175(12):1895-6. doi: 10.1001/ jamainternmed.2015.5689. Review.

19.Kairosweb. Precio al 30 de abril de 2017 de la vacuna Prevenar 13 (Vacuna antineumocócica conjugada 13-valente) 1054,22 pesos argentinos [Internet: fecha de último acceso 02/06/17]. Disponible en: http://ar.kairosweb.com/laboratorios/producto-prevenar-13-21229

20. Kairosweb. Precio al 22 de mayo de 2017 de la vacuna Pneumo 23 (Vacuna antineumocócica polisacárida 23-valente) 534,94 pesos argentinos [Internet: fecha de último acceso 02/06/17]. Disponible en: http://ar.kairosweb.com/laboratorios/producto-pneumo-23-4934

21. Argentina. Ley Nacional $N^{\circ} 22.909$ [Ley de vacunación obligatoria] (Septiembre 13, 1983) [Internet: fecha de último acceso 02/06/17]. Disponible en: http://servicios.infoleg. gob.ar/infoleglnternet/anexos/45000-49999/48717/norma.htm

22. Gentile Á, Abate H. A new challenge for the world: the eradication of polio. Arch Argent Pediatr. 2016 Dec 1;114(6):557-562. doi: 10.5546/aap.2016.eng.557. English, Spanish.

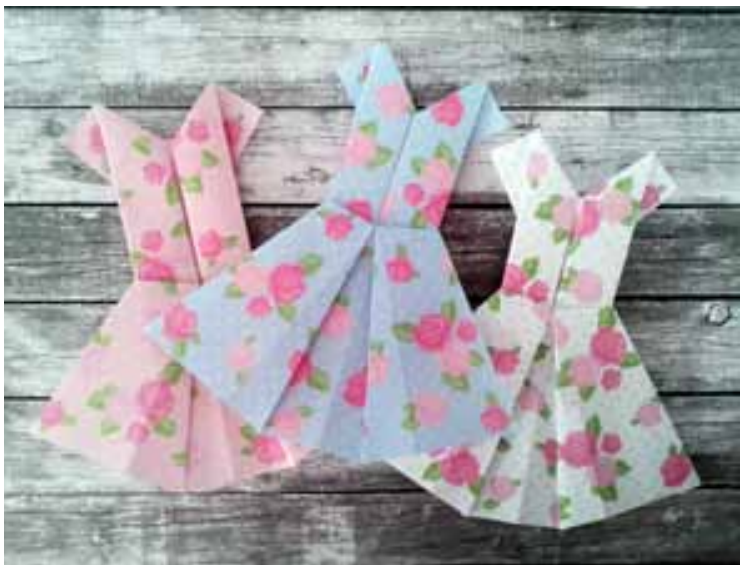

Gentileza: Foto y plegados de Claris Viviano (www.origamialalma.com) 